RUNNING HEAD: Aging brain structure and function

\title{
Structure and function of the aging brain
}

\author{
R. Nathan Spreng ${ }^{1,2} \&$ Gary R. Turner ${ }^{3}$
}

${ }^{1}$ Laboratory of Brain and Cognition, Montreal Neurological Institute, Department of Neurology and Neurosurgery, McGill University, Quebec, Canada.

${ }^{2}$ Departments of Psychiatry and Psychology, McGill University, Quebec, Canada

${ }^{3}$ Department of Psychology, York University, Toronto, ON, Canada

Spreng, R.N., Turner, G.R. (2019, forthcoming) Structure and function of the aging brain. In G Samanez-Larkin (Ed.) The aging brain. Washington DC: American Psychological Association.

\section{Corresponding Author:}

R. Nathan Spreng

Laboratory of Brain and Cognition

Montreal Neurological Institute

Department of Neurology and Neurosurgery

McGill University, Quebec, Canada

e: nathan.spreng@gmail.com 
Aging brain structure and function

\section{INTRODUCTION TO THE AGING BRAIN}

In this opening section of the Aging Brain we set the stage for the contributions that follow by providing a broad overview of the latest advances in our understanding of how the brain changes, both structurally and functionally, across the adult lifespan. We leave domain-specific aspects of brain aging to the subsequent chapters, where contributors will provide more targeted accounts of brain change germane to their particular focus on the aging brain. Here we review the extant, and rapidly expanding literature to provide a brief overview and introduction to structural and functional change that occur with typical brain aging. We begin the chapter by looking back, to review some of the early discoveries about how the brain changes across the adult lifespan. We close the chapter by looking forward, towards new discoveries that challenge our core assumptions about the inevitability or irreversibility of age-related brain changes. These sections serve as bookends for the core of the chapter where we review the latest research advances that continue to uncover the mysteries of the aging brain.

A brief history of brain aging. Whether in the lab or in the clinic, we now take for granted the ready access to measurement tools, and the precision with which we are able to investigate lifespan changes in the structure and functioning of the human brain in vivo. However, the study of the aging brain a relatively young endeavor. Early neuroscientific studies did not include, or perhaps even consider, systematic investigations of brain changes that may occurr in later life. Indeed, a leading text on the history of neuroscience, Origins of Neuroscience [1], does not include a section on older adult brain development and the term 'aging' does not even appear in the subject index. Of course this may be explained in part by the comparatively restricted range of the human lifespan before the turn of the $19^{\text {th }}$ century. However, by the mid $20^{\text {th }}$ century, researchers in the fields of medicine, neuroscience and evolutionary biology began to recognize that the brain does not remain stable across the normal adult lifespan. Post-mortem investigations began to report both grey and white matter volume loss as well as ventricular enlargement in older versus younger adults [for an early review see 2]. However, these early pathological and comparative neurological studies were plagued by small sample sizes and methodological constraints that impacted measurement reliability [3]. By the latter decades of the $20^{\text {th }}$ century, post-mortem studies also began to identify broad topological patterns of age-related change in brain structure. Sensory cortices were seen be comparatively preserved while more pronounced changes were evident in association cortices, including frontal and lateral parietal regions [4]. Following rapidly from these post-mortem investigations, in vivo neuroimaging techniques, including CT and 2D MRI, became more widely adopted. These methods were critical in advancing our understanding of the aging brain as they allowed the enterprise of brain research to essentially 'scale up', enabling the collection of brain volume measures from larger groups of participants that could then be more reliably compared across age cohorts. These imaging techniques, and in particular the development of high resolution, 3D MRI neuroimaging methods in the late 1980s and early 1990s, as well as more sophisticated registration protocols necessary to spatially align individual brains to conduct group comparisons, opened the way for larger cohort and longitudinal studies that have become standard in the field today [5].

As with studies of structural brain aging, the earliest investigations of aging brain function in humans emerged in the middle decades of the $20^{\text {th }}$ century. Indeed, as early as 1938 electroencephalogram (EEG) recordings were seen as offering 'appreciable promise as a means to characterize significant deviations from the 'natural' aging found in Alzheimer and other dementias' [Berger, 1938, as reviewed by 6]. In the latter part of the century, increasingly sophisticated methods emerged to investigate age-related changes in brain function. These included single photon emission computed tomography (SPECT), positron emission tomography (PET), functional neuroimaging (fMRI) and magnetoencephalography (MEG) techniques. In the early decades of the $21^{\text {st }}$ century additional techniques including intracranial electroencephalography (iEEG) or electrocorticography $(\mathrm{ECoG})$ methods have enhanced the spatial and temporal resolution 
Aging brain structure and function

of functional brain measurements. Transcranial magnetic stimulation (TMS) and transcranial direct current stimulation (tDCS) techniques now allow for the temporary modulation of brain activity, enabling researchers to more directly investigate how changes in brain function are associated with cognitive functioning in later life [e.g. 7, 8]. AS we will see at the end of the chapter, these brain stimulation techniques are also offering considerable promise as interventions or treatments to potentially alter the course of cognitive aging [e.g. 9].

In the following sections of the chapter we will survey findings drawn from each of these techniques to provide a comprehensive overview of the current state of research on structural and functional brain aging. As we have seen, the study of the aging brain remains in its infancy. Brain imaging, allowing us to measure and record from the brain in vivo, is barely half a century old. Yet despite this brief history, much is now known about the myriad ways in which our brains change as we move through adulthood and into older age - and of course much remains to be discovered.

\section{STRUCTURAL BRAIN CHANGES IN OLDER ADULTHOOD}

Age-related changes in the structure of the brain have been examined across multiple, interacting levels, from cells and synapses to regions or structures and, more recently, large-scale brain systems or networks. While structural brain changes measured using in vivo techniques almost certainly reflect cellular changes including dendritic branching, synaptic density and demyelination of local and long-range axonal fibers, here we focus our review on measures of parenchymal (grey and white matter) and cerebrospinal fluid volumes, both globally and regionally, and how local changes converge at the level of whole-brain networks. Consistent with the vast majority of research literature in this field, we also limit our primary focus to changes primarily involving cortical structures. However, age-related changes to subcortical structures are of increasing interest in understanding age-related changes in cognitive and affective behaviors [e.g. 10] or as early markers of atypical brain aging [e.g. 11] and we highlight these where possible throughout our review. While earlier studies measured structural change almost exclusively in terms of volumetrics; more recent work has emphasized other features, including topological variability, rates of change and inter-individual differences. In this section we provide an overview of the changing structure of the brain in older adulthood at each level of analysis and across these multiple features of brain aging. While not the primary focus of the chapter, this evolving, multidimensional perspective on structural brain changes in older adulthood is gaining prominence in the search for sensitive neural biomarkers that signal transition from normal aging to brain disease, a topic we discuss briefly in the final section.

\subsection{From post-mortem to in vivo: Early findings}

Early in vivo neuroimaging studies of structural brain aging focused primarily on global changes in tissue compartments including grey and white matter and cerebrospinal fluid volumes [see 12, for a review]. These studies confirmed post-mortem investigations that had reported global volume loss and ventricular enlargement in older relative to younger adults [2]. In addition to confirming these ex vivo findings, early neuroimaging investigations provided the first indication of topological variability, with changes occurring at different rates for different brain regions and tissue types. This pattern, described as a 'patchwork pattern of differential declines and relative preservation' [13], suggested that not all brain regions demonstrate a similar extent or rate of decline across the lifespan. As we will see below, more recent work suggests an even more complex picture of structural brain aging, with different regions demonstrating significantly different, and often non-linear, trajectories of change (Fjell, 2014).

Investigations of structural brain aging using in vivo, neuroimaging methods began to increase rapidly towards the end of the last century as MRI technology became more readily accessible and numerous groups began efforts to map the trajectory of age-related brain changes 
Aging brain structure and function

with increasing topological specificity. However, these efforts were not without controversy. Many of the earliest in vivo studies adopted a cross-sectional approach, comparing measures of brain structure between younger and older adults. However, cross-sectional designs have been criticized for providing purely chronologically-based estimates of brain age. In other words measurements at a single time-point can only characterize brain structure at that time point. Single-point measures are unable to describe age-related changes in brain structure [14]. There are two primary criticisms of cross-sectional designs. First, they are contaminated by cohort effects, which confound group membership and age in difference calculations. Second, cross sectional designs do not account for individual differences which introduce significant variability in group level estimates, and likely deflate true estimates of structural brain change between age groups. With the increasing accessibility of MR technology, research groups began to conduct longitudinal studies of agerelated changes in brain structure. While complex and costly, longitudinal studies hold the advantage of accounting for individual differences and controlling for cohort effects, providing what is arguably a truer estimation of age-related brain change. As a reflection of the differences in these two approaches, a recent study of age-related cortical thinning reported annualized rates of $-0.30 \%$ using cross-sectional methods. This estimate stands in somewhat stark contrast to the estimated annualized rate of $-0.59 \%$ using a longitudinal study methods [15]. The difference represents a nearly two-fold difference in annual change estimates. These results are consistent with an earlier study that used a combined cross-sectional and longitudinal design [16] and again reported deflated cross-sectional change estimates. Taken together these studies urge caution in the interpretation of these estimated structural brain changes. While cross-sectional studies of agerelated brain change may be more feasible, cost-effective, and allow for larger study samples to be collected, these designs may nonetheless under-estimate the magnitude of age-related brain changes.

Since the earliest in-vivo investigations, the number of studies investigating structural brain changes associated with normal aging has increased exponentially. Taken together the findings provide a complex picture, with often conflicting findings. In the following sections we review the extant literature and distill the findings into the most commonly reported and replicated patterns of structural brain change in older adulthood. We begin by reviewing the most recent evidence for global changes in whole-brain and ventricular volumes and then summarize the current state of knowledge with respect to region-specific trajectories of change. The most common in vivo metrics for measuring changes in brain structure are volumetrics, cortical thinning, and surface area and we limit our review almost exclusively to these measures. As the health of cerebral white matter is assuming a place of increasingly importance in the study of brain and cognitive aging, we review patterns of white matter change, including volumetrics, white matter integrity, and lesion burden in a separate section. Finally, we end this section of the chapter on structural brain aging with a brief review of structural brain networks. These covarying patterns of structural brain change appear to be potent predictors of the transition from normative to diseased aging, and may in fact identify disease-specific structural network biomarkers.

\subsection{Global and regional changes}

Global changes. Age-related changes in cerebral parenchyma and ventricular volumes have been reported from the earliest in vivo studies [reviewed in 12]. Interestingly, while grey matter, CSF and ventricular volume changes were consistently reported in these early studies, agerelated changes in white matter volumes were generally not observed, although microstructural changes in white matter had been reported previously [17]. Age-related changes in whole brain volumes were also reported in one of the first whole-brain, volume-based morphometry (VBM) studies, with evidence that global grey matter volumes declined linearly and CSF volume increased linearly with age [3]. Again, consistent with earlier reports, no age-changes were observed in global white matter volumes. In contrast, white matter volume changes were reported in an early 
longitudinal study from the Baltimore Longitudinal Study of Aging [18]. Age-related declines on the order of 5.4, 2.4 and $3.1 \mathrm{~cm}^{3}$ per year were reported for total brain, grey, and white-matter volumes, respectively, while ventricular volume increased by $1.4 \mathrm{~cm}^{3}$ per year. These findings were recently replicated in a larger sample from the same study cohort [19].

Longitudinal investigations have characterized global (and regional) changes in brain structure in terms of annualized percent change estimates. Annualized percent change is a standardized metric for determining the rate and trajectory of age-related changes by calculating a per year estimate of decline. Annualized percent change is typically calculated voxel-wise (VBM) or vertex-wise (cortical thickness) for each participant across multiple timepoints, thus allowing for both global and regional estimates of change. While different formulae for calculating annualized percent change have been reported, a typical approach involves calculating differences in volume (or thickness) between time points which is then divided by the baseline volume (or thickness) estimate and the number of years between time points [e.g. 20]. Longitudinal studies of cortical thickness have reported annualized percent change ranging from -0.59 in a cohort with mean age of 75 [15] to -0.35 for lifespan [21] and young-old (60-66) samples [20]. In one of the few non-Western studies reported in the literature, annual change of $-0.56 \%$ for total brain volumes were observed in the Singapore-Longitudinal Aging Brain Study [22]. These findings provide strong evidence that declines in parenchymal volume, and concomitant increases in CSF and ventricular volumes, are a hallmark of adult aging. Despite earlier cross-sectional reports, longitudinal studies demonstrate convincingly that both grey and white matter tissue compartments decline with age. While estimates and measures vary widely across studies, there appears to be convergence around the extent of parenchymal change, with annual loss estimates ranging from 0.30 to -0.56 percent per annum. Further, this rate of global decline appears to accelerate in late life from young-old (60-66 years) [20] to middle-old ( $\sim 75$ years) [15] and old-old ( +90 years) [23]. Finally, while a full review of sex differences in age-related brain change is beyond the scope of this review, it is important to note that the majority of published reports (whether cross-sectional or longitudinal) and across both global and regional measures, show a steeper and more rapid trajectory of decline for males than females [e.g. 3, 19, 20, 24, 25-27]

Regional changes. The earliest in vivo imaging studies reported global, or non-specific, brain changes in late life [28]. However, evidence from animal and human post-mortem studies [2, 4] and later in vivo imaging studies [e.g. 26, 29] observed regional variability in structural brain changes occurring in late life. Indeed, the extent of regional variability was described in these early studies as a 'patchwork' of age-related change across the cortical mantle [26]. This distributed or 'heterochronus' [5] nature of structural brain changes across the lifespan has been observed repeatedly in both cross-sectional [5, 23, 30-34] and longitudinal [18-21, 27, 35-37] studies.

In a targeted investigation of regional differences in brain atrophy using region of interest (ROI) methods, the most robust age changes were observed in prefrontal gray matter [26]. The authors interpreted this finding in the context of earlier post-mortem and in vivo imaging studies, suggesting that anterior brain regions, particularly prefrontal and temporal regions may undergo accelerated changes with age [see 12, for a review of these early studies]. Subsequently, longitudinal studies appeared to confirm this finding of greater susceptibility of prefrontal and temporoparietal association cortices to age-related decline $[5,15,18,20,35,36]$. While we discuss network level changes in more detail below, several studies have specifically identified regions of the default network, a collection of functionally interconnected brain regions situated primarily along the brain's midline, as showing increased susceptibility to age-related changes $[21,35]$. Explanations for these regional atrophy patterns include the 'last-in, first-out' hypothesis [13], suggesting that brain regions such as the prefrontal cortex, which reach full maturation later in life, may be the most vulnerable to early decline in late adulthood. Similarly, the extended development - sensory hypothesis suggests that all heteromodal association cortices atrophy earlier, followed by declines in primary sensory-motor and paralimbic cortices in later older adulthood [38]. 
Aging brain structure and function

On balance, existing research is consistent with these hypotheses, with frontal and heteromodal association areas most commonly identified as undergoing more rapid decline than sensory-motor regions $[16,19,21,25,39]$. This leads directly to the conclusion that accelerated volume loss is not simply a global feature of structural brain aging. Support for this idea was recently provided in a large cross sectional study, with a smaller longitudinal validation cohort [35]. Brain regions followed one of several trajectories with critical change periods, or inflection points, occurring in late adolescence or middle adulthood. Structures including the hippocampus, brain stem regions, cerebellar and cortical white matter, showed stability (or increases) in cortical thickness, followed by steep declines in later life. Structures including the amygdala, putamen, thalamus, nucleus accumbens, and cerebellar cortex showed a pattern of near linear decline across the lifespan. A third category, which included estimates of global parenchymal and cortical volumes, followed a quadratic function with accelerating decline in later life [35]. Regional variability in rates of brain atrophy was also reported in two recent studies of young-old [20] and old-old [23]. In young-old, greater annualized percent change was observed in heteromodal than in primary sensory motor cortices, with inferolateral temporal and inferior parietal cortices showing particularly pronounced changes. In contrast, for old-old adults, accelerated changes were observed in medial temporal and occipital cortices, particularly in the $10^{\text {th }}-11^{\text {th }}$ decades of life [23]. Structural declines have also been reported in primary sensory motor and occipital brain regions in old-old adulthood [5, 21]. Together these findings are consistent with the 'retro-genesis' hypothesis [38], with prefrontal and heteromodal cortices developing later and declining earlier than primary sensory-motor regions.

\subsection{White matter changes}

A recent post-mortem investigation of structural brain changes in older adulthood reported reduced cerebral white matter volume in both anterior and posterior brain regions while failing to find age-related changes in cerebral grey matter [40]. These results prompted the study authors to suggest that 'healthy brain aging is a process affecting predominantly white, not grey, matter'. These findings hint at the importance of considering white matter changes as a core feature of structural brain aging. Interestingly, changes in global white matter volume were not commonly reported in early cross-sectional studies, although subsequent well-powered cross-sectional and longitudinal studies did report reliable age-related declines in overall white matter volumes $[16,18$, $25,41]$. Consistent with reports of regional specificity in cortical volume and thickness changes, white matter changes also appear to follow an anterior-posterior gradient with the most rapid atrophy occurring in frontal white matter $[16,42,43]$. The vast majority of reports of white matter atrophy also describe a curvilinear pattern, with more rapid declines occurring in later older adulthood [23, 25, 35, 41, 44].

Beyond volumetrics, white matter changes have also been characterized using diffusion imaging methods to assess the integrity of white matter fibre tracts in the brain. Several recent reports suggest that changes in white matter integrity may precede grey matter changes, thus providing a more sensitive marker of structural brain changes in normal aging [45, 46]. Declines in white matter integrity have now been reported across numerous studies and again appear to follow an anterior to posterior gradient with the most rapid changes occurring in frontal white matter compartments [47, 48]. A third metric for characterizing the health of cerebral white matter involves measuring the volume of white matter lesions. Lesions in the brain's white matter are thought to occur as a result of small cerebrovascular events, leading to alterations in axonal myelin and ultimately membrane permeability resulting in axonal damage. White matter lesions are associated with cerebrovascular risk factors [49] and, given increasing rates of obesity and metabolic diseases in Western populations, likely represent one of the most rapidly growing forms of structural brain change in older adulthood. White matter lesion burden appears to rapidly increase in the oldest old with one recent report suggesting decelerating volume loss and 
Aging brain structure and function

accelerating lesion volumes in this cohort [23]. Further the presence of cerebral small vessel disease, including artherosclerosis, deep white matter lesions, or subcortical lacunar infarcts is strongly associated with Alzheimer's disease [50] and a nearly two-fold increased risk of dementia onset [51].

\subsection{Changes in structural brain networks}

Over the last decade research investigating structural brain change in older adulthood has expanded beyond global and regional changes to consider distributed patterns of structural decline. Structural covariance is observed as inter-individual differences in regional brain structure covarying with other brain structures across the population [52-54]. Across individuals, intrinsically connected functional brain networks, such as the default network, can be topographically represented in the structural patterns of cortical gray matter. Patterns of covariance in brain structure were first identified in post-mortem studies [55], and changes in structural covariance networks with age have now been reported in whole-brain in vivo studies [56-63].

As with global and regional measures of structural brain changes, structural covariance changes with age are more prominent between frontal brain and posterior cortices, reflecting a loss of long-range covariance in favor of increased local processing [61, 63, 64]. Another prominent feature of network-level changes is declining structural covariance within the default network, a collection of functionally-connected brain regions implicated in mnemonic and associative processing [65]. In a recent report, structural covariance patterns were identified from seed regions showing maximal atrophy across various neurodegenerative diseases. Structural covariance with these seed regions in young adults reflected atrophy patterns in a disease specific manner [66]. As one example, structural covariance of the default network in young showed high spatial coherence with the pattern of neurodegeneration observed in Alzheimer's disease. We recently reported similar patterns of reduced structural covariance within the default network, with changes observable over as little as two years in a normal aging cohort. Further, we observed that changes in default network structural covariance reliably predicted the transition from mild cognitive impairment to Alzheimer's disease in a longitudinal sample [62]. Similar patterns of declining structural covariance have been reported in other large-scale distributed brain networks, including executive control networks, which have been implicated in those cognitive functions most susceptible to aging [61]. Indeed, measures of structural covariance, when combined with estimates of cerebral blood flow, explained almost all age-related variance in cognitive performance in a recent report [67]. This last observation speaks to the importance of measuring not simply independent trajectories of regional changes, but covariance patterns describing how volume changes in distributed brain regions cohere across the across the lifespan.

\subsection{Summary of structural brain changes in aging}

With the advent of increasingly powerful in vivo neuroimaging techniques, the study of age-related structural brain changes represents a vast and expanding field. Given the scope of the review, we chose to focus on the broad trends that have been most reliably reported over the last two decades of structural neuroimaging research. Global changes in grey matter, white matter and ventricular volumes are clearly a hallmark of normal brain aging. Heteromodal association cortices

are more susceptible to late life structural decline than primary-sensory motor regions, and rates of decline differ among cytoarchitectonic zones. Rapid declines occur in frontal and heteromodal association cortices in young-old, with comparatively shallow rates of decline observed in medial temporal lobe and primary sensorimotor regions. This is followed by more rapid changes in the medial temporal lobe and primary sensorimotor motor regions in old-old adulthood. White matter changes, whether measured as volume, integrity or lesion burden, are also a prominent feature of brain aging and may in fact be a stronger predictor of cognitive decline and dementia than cortical 
changes. Finally, investigators are increasingly moving beyond region-specific metrics to identify whole brain patterns of structural brain change. Measures of structural covariance have proven to be powerful predictors of cognitive capacity in normal aging and as potent biomarkers of the transition from normal aging to neurodegenerative disease. Understanding these normative patterns of structural brain change is critical to expanding opportunities for detecting non-normative brain aging using in vivo imaging methods. However, as we noted in our chapter introduction, age-related brain change is multi-dimensional, impacting both structure and function. In the next section we turn attention to changes in how the brain functions in older adulthood again considering how these are reflected globally, regionally, and at the level of interacting networks.

\section{FUNCTIONAL BRAIN CHANGES IN OLDER ADULTHOOD}

Functional neuroimaging methods have been used to study the aging brain for over three decades. Much of the work over this period has utilized these methods to identify the neural correlates of cognitive functioning across a myriad of domains (e.g. sensory-motor, processing speed, memory, executive function). As with the research literature on structural brain changes, the findings characterizing functional changes in older adulthood are often varied and provide conflicting perspectives as to the nature and implications of observed differences in brain activation between age cohorts. In this section we briefly review domain specific brain changes, focusing on the findings of our recent meta-analytic reviews. We then review more domain-general patterns of functional brain changes in older adulthood and describe several of the leading theoretical perspectives in the field. As with our review of structural brain aging, we end this section by describing age-related functional brain changes at the level of large-scale, functionally connected brain networks. We have chosen to focus here on functional neuroimaging studies using MRI or positron-emission tomography (PET) measures of brain function. While much research and numerous advances have been made using electrophysiological techniques, including EEG and MEG studies, we are unfortunately unable to cover these techniques within the scope of the review.

\subsection{Domain-specific changes}

The field of neurocognitive aging research has rapidly expanded over the last two decades. While a comprehensive survey of the literature across this vast literature is beyond the scope of this one chapter, we have published three meta-analytic reviews of studies investigating age-related functional brain changes. The first meta-analysis included 80 functional neuroimaging studies across four cognitive domains: perception, memory encoding, memory retrieval and executive functioning [68]. For perceptual tasks, older adults showed greater dorsolateral PFC as well as anterior insula and frontal opercular activation, whereas younger adults showed the predicted pattern of greater activity in sensory cortices, particularly occipital regions. For memory tasks young adults showed greater right lateral PFC and medial temporal activity during encoding whereas older adults preferentially engaged right PFC regions during memory retrieval. Agedifferences during executive control tasks were primarily observed in prefrontal brain regions. Specifically, older adults showed greater activation in more dorsal aspects of PFC bilaterally whereas younger adults showed greater recruitment of right ventrolateral PFC regions. Across all domains, older adults engaged prefrontal regions to a greater extent than young adults. In contrast, younger subjects, particularly those showing poorer task performance, engaged posterior sensory regions. Further, the enhanced pattern of PFC recruitment observed in the older adult cohorts was

performance-dependent. Higher performing older adults showed greater left lateralized prefrontal engagement while lower performing subjects engaged regions of right PFC.

In two follow-up meta-analyses we examined age-differences specifically in the domain of executive control processing. We examined patterns of age-related brain change associated with discrete executive control processes including working memory, inhibition and task switching [69, 
Aging brain structure and function

70]. Consistent with the findings of the earlier review, we observed a general pattern of increased functional brain activity for older versus younger adults. However, the specific nature of this enhanced functional recruitment was process specific. Task-switching and working memory were associated with increased prefrontal recruitment bilaterally. In contrast, inhibition showed a 'young-plus' pattern with age-related increases localized to regions typically implicated in young. Again, the most robust age difference observed across all three control processes was enhanced recruitment of prefrontal brain regions for older versus younger adults. This age-related difference in PFC activity was greater at higher levels of working memory demand, suggesting that increased recruitment of these regions may reflect greater reliance on, or strategic engagement of, working memory resources in older adulthood [69].

\subsection{Domain-general changes: Neural dedifferentiation}

As reviewed above, early functional neuroimaging studies of cognitive aging typically adopted a domain-specific approach, with investigators enumerating age-related changes in the neural implementation of specific cognitive task performance using cross-sectional study designs [for reviews see 68, 70, 71, 72-77] Taken together, these studies also identified domain-general patterns of functional brain changes in aging, suggesting that all age-related cognitive changes may share, at least in part, a common neural substrate. Perhaps the most ubiquitous pattern observed across studies has been referred to as neural dedifferentiation, increased and more spatially distributed patterns of neural activity in older versus younger adults during cognitive task performance [78]. In one of the earliest functional neuroimaging investigations of cognitive aging, PET scanning methods were used to measure changes in metabolism across brain regions while younger and older participants performed visuoperceptual tasks. Older participants displayed greater functional activation during task performance than younger participants. Moreover, unlike the lateralized pattern of functional activity within the prefrontal cortex observed in the young, older participants demonstrated greater bilateral activation [79]. Since this seminal work, this pattern of decreased lateralization in functional brain response in aging has been replicated in numerous reports using both PET and fMRI methods, and spanning a range of cognitive domains including memory encoding and retrieval [80-84], visual attention [80-85]; working memory [8084, 86-89], as well as selective attention and inhibition [90].

The finding that cortical activation patterns become increasingly differentiated has become a leading account of functional brain changes in older adulthood and indeed of neurocognitive aging. Dedifferentiation has been operationalized in a number of ways. It has been described simply as non-identical brain activity patterns between younger and elder populations [91] or as more diffuse and distributed cortical representations of cognitive activities [92]. Other researchers suggest that it reflects a failure to engage specialized neural mechanisms during cognitive performance [81, 93]. Three forms of dedifferentiation have been described [78]. Contralateral recruitment refers to the age-related recruitment of brain regions homologous to those recruited in younger participants [e.g. 94]. Unique recruitment describes the engagement of additional (nonhomologous) brain regions [e.g. 83, 85, 95]. Finally, substitution reflects activation of entirely novel neural networks in older relative to younger adults, perhaps signaling strategy differences or functional reorganization [e.g. 96, 97].

Evidence for dedifferentiated neural response in older versus younger adults suggests that reduced neural specialization may provide a neural marker of age-related cognitive decline. However, as a theory of neurocognitive aging, it is ambivalent with respect to whether these brain changes are compensatory or deleterious. In other words, does dedifferentiated neural response reflect compensatory functional responses or inefficient processing in older adults? In general, decreased lateralization of functional brain activity (i.e. greater dedifferentiation) has been considered compensatory for cognitive performance in older adulthood. In an early report, older participants who performed better on a verbal memory task showed greater bilateral PFC activation 
than those who performed more poorly, suggesting that dedifferentiated neural activity was indeed a compensatory functional response to degraded neural circuitry in healthy aging [81]. Dedifferentiation through substitution has also been positively associated with cognitive performance. During an incidental encoding task, older adults recruited medial temporal lobe regions less, and lateral PFC regions more, than their younger counterparts [98]. Moreover, recruitment of lateral PFC and medial temporal lobe structures were inversely correlated in older but not younger participants. The investigators concluded that since the analysis was only conducted on 'remembered' stimuli, dedifferentiation was compensatory for recognition performance in the older adults. Perhaps the most compelling evidence that dedifferentiation is compensatory was provided by the application of repetitive transcranial magnetic stimulation (rTMS) to older and younger participants during an episodic memory task. While memory retrieval was disrupted by rTMS to a right PFC region in younger participants, older participant performance was disrupted by rTMS applied to both right and left PFC, suggesting that greater bilateral recruitment (i.e. dedifferentiation) supported cognitive performance in these participants [8].

In contrast to these compensatory accounts, several functional neuroimaging studies have reported that dedifferentiated neural response is associated with poorer, not improved, cognitive ability in older adults. In one of the few studies to directly contrast these competing behavioral accounts of neural dedifferentiation, functional compensation and neural inefficiency hypotheses were directly contrasted in a sample of healthy older and younger adults during performance of a delayed recognition task [91]. The authors observed evidence of inefficient neural responding (i.e., greater activity for equivalent performance) in older relative to younger participants across a large area of cortex during encoding and maintenance epochs of the task. Moreover, the spatial patterns of response in younger participants were more similar to the pattern observed in higher performing than lower performing older adults; inconsistent with a compensatory account.

Dedifferentiation of neural response in older relative to younger adults has been one of the most ubiquitous findings in the neurocognitive aging literature. Moreover, this account of functional brain changes parallels a similar pattern of dedifferentiation in behavioral performance across cognitive domains in older adulthood [99]. While there is strong empirical evidence demonstrating dedifferentiation of functional brain response in older adulthood, the data remains equivocal as to whether these changes are compensatory, or associated with cognitive decline in later life. In the next section we briefly review several leading theories of age-related changes in brain function that attempt to reconcile the compensation versus decline debate, while also providing more specific accounts of the topology and cognitive implications of age-related functional brain changes.

\subsection{Theories of brain function in older adulthood}

As with studies investigating structural brain changes, accounts of functional change across the adult lifespan are highly variable and report somewhat conflicting findings with respect to the patterns of change and their implications for cognitive function in later life. However, areas of broad convergence have emerged and these have been characterized by several leading theories of neurocognitive aging.

Consistent with the evidence for functional dedifferentiation reviewed above, a series of studies investigating brain changes during episodic, working memory and visual attention tasks, older adults demonstrated a robust pattern of reduced asymmetry in the pattern of activation across cerebral hemispheres [80]. This pattern of overlapping, or dedifferentiated, neural response across cognitive tasks was described as Hemispheric Reduction Asymmetry in Older Adults (HAROLD). Older adults demonstrating the HAROLD pattern of functional brain changes showed better performance on an episodic memory task than those who showed a more 'young-like' pattern of asymmetry in the recruitment of prefrontal brain regions during the task, suggestive of 
compensation. Further, this pattern was observed across multiple cognitive domains including episodic memory, working memory and visual attention [100].

Age-related functional brain changes have also been observed in response to increasing levels of task challenge leading to the Compensation-Related Utilization of Neural Circuits Hypothesis (CRUNCH) of cognitive aging [76, 101]. This theory posits that inefficiencies in neural processing may cause older adults to over-recruit neural resources to achieve the same level of cognitive performance as younger adults. As with HAROLD, increased, or dedifferentiated, recruitment patterns were seen as evidence for compensatory activity, necessary to overcome degraded or noisy neural signaling associated with broader neuronal tuning curves [e.g. 102] or degraded signaling pathways [e.g. 47]. The CRUNCH hypothesis predicts two patterns of functional brain change that are commonly reported in older adulthood. At lower levels of task demand, increased recruitment is observed in the context of equivalent cognitive performance for older and younger adults. However, as task demands increase, older adults demonstrate lower levels of brain activity than young and task performance declines. Thus, while considered a compensatory account, by incorporating levels of task demand CRUNCH suggests that older adults show poorly modulated and inefficient neural recruitment patterns, with greater brain activity required per unit of cognitive output.

A third theory of neurocognitive aging integrates both structural and functional brain changes when considering the behavioral implications of dedifferentiated patterns of brain activity in older adulthood. The Scaffolding Theory of Aging Cognition (STAC) argues that changes in cortical volume, white matter integrity and neurochemical signaling may be counteracted, at least in part, by the construction of neural 'scaffolds' $[75,77]$. Conceptually similar to the CRUNCH hypothesis, these 'scaffolds' involve the functional recruitment of additional neural resources to offset these age-related structural brain changes. In this model, dedifferentiated patterns of brain response in older adulthood reflect a scaffolding process wherein additional neural resources are engaged to supplement task-specific recruitment patterns observed in younger adults[77]. As reviewed in the domain-specific section above, these scaffolds, or patterns of enhanced recruitment, in older adults typically involve activation of anterior brain regions bilaterally, consistent with the Posterior to Anterior Shift in Aging (PASA) hypothesis [95] and the HAROLD models [80].

The final theory we will review in this section on domain general theories of functional brain aging is neuromodulation. This account of age-related functional brain changes argues that declines in the goal-directed modulation of neural activity is a central mechanism of neurocognitive aging $[103,104]$. Consistent with this idea, reduced selectivity in neural responses in categoryselective regions of visual association cortex in older relative to younger participants have been reported during a working memory task [105]. Critically, this reduced selectivity in neural responses (i.e. noisier processing) was accompanied by enhanced activity in the PFC, suggesting greater PFC activity was necessary for older adults to modulate visual association regions in response to degraded perceptual representations. A similar pattern of age-related deficits in the modulation of neural responses based on task goals has been reported during selective working memory [103]. During the task, age-related reductions in goal-directed suppression of activity in the visual association cortex resulted in poor filtering of goal-irrelevant stimuli and, critically, these brain changes predicted subsequent impairments on a recognition memory paradigm.

Consistent with this idea, impaired modulatory capacity, as seen in older adulthood, has been shown to attenuate neural responsiveness to afferent signaling in posterior brain regions, producing poorly regulated and noisy information processing as evidenced both in computational modeling [102] and functional neuroimaging [106] studies. Resultant reductions in signal-to-noise ratios degrade the integrity of mental representations, thus reducing the quality of information throughput to higher cognitive processes. According to the neuromodulation account, reduced modulatory capacity should preferentially impact those domains dependent upon the highest levels of representational complexity, including episodic memory, selective attention, and working 
Aging brain structure and function

memory. These are indeed among the most vulnerable to age-related decline, and show robust patterns of dedifferentiated brain response in prefrontal brain regions [c.f. 68, 69, 70].

\subsection{Changes in functional brain networks}

With the advent of whole-brain, in-vivo functional neuroimaging methods, and recent advances in computational resources and multivariate analytical methods, neurocognitive aging is increasingly studied through the lens of large-scale functional brain networks [107]. Commonly reported patterns of age-related changes in neural networks, or functionally connected assemblies of spatially distributed brain regions, include changes occurring within specific brain networks, as well as alterations in the dynamic interactions among networks.

Investigations of network changes associated with normal aging have typically implicated the default network, a collection of functionally connected brain regions including the posterior cingulate cortex (PCC), medial PFC (MPFC), inferior parietal lobule as well as medial and lateral temporal lobes [70, 108-114]. The default network is activated during social or internally-directed cognitive processes, including access to stored knowledge representations and experiences [65] and is typically suppressed during performance of externally-directed tasks [115]. Age-related changes include reduced suppression $[112,116,117]$ and decreased within-network connectivity during both task $[110,113,118]$ and rest $[108,109,119,120]$. Recent evidence also suggests that the default network is more functionally connected to other brain networks in aging [113, 114, 119$122]$ and this connectivity is poorly modulated by task context [96, 97, 122-124].

While changes involving the default network have been frequently reported, there is mounting evidence to suggest that the global network architecture of the brain is altered across the adult lifespan. This has been characterized as reduced network segregation and increased integration [119]. As with patterns of brain activity (reviewed above), interactions among spatiallydistributed brain networks become increasingly dedifferentiated in older adulthood. In the context of functional brain networks, this means that with age, interactions between networks increase (i.e. they become less segregated or differentiated), while within-network connectivity declines. Measured across the whole brain, older adults display a less discrete network architecture both during cognitive task performance $[119,125]$ as well as during rest, suggesting these functional network changes are also manifest within the intrinsic network architecture of the brain [e.g. 120].

Similar patterns of network differentiation with age have been reported in a more circumscribed set of brain networks, including the default, dorsal attention and frontal parietal control networks both during task and at rest $[122,123]$. Specific changes include reduced anticorrelations between dorsal attention and default networks and increased network interactions across all three networks, consistent with a network dedifferentiation account. We have also reported poor modulation of network interactions based on task goals. Older adults fail to decouple default and frontoparietal control networks in response to changing task context [124] and control demands [97]. These observation led us recently to propose the Default-Executive Coupling Hypothesis of Aging [DECHA, 97]. This network neuroscience model of neurocognitive aging suggests that with age, older adults fail to flexibly decouple brain regions implicated in control processes from the default network, implicated in more associative cognitive processes. We have recently shown that increased coupling of these networks, as predicted by the DECHA is associated with reduced fluid intelligence and increased reliance on semantic or crystalized knowledge in older adulthood [126]. Recent evidence both from cross-sectional [96] and longitudinal [127] investigations provide support for this hypothesis and provide further evidence that these changes in network interactivity can predict individual differences in cognitive functioning. Taken together these network-based accounts of functional brain aging point to network neuroscience as an important new frontier in neurocognitive aging research.

\subsection{Summary of functional brain changes in aging}


Aging brain structure and function

In this section we have reviewed the current state of knowledge with respect to changes in brain function that occur from younger to older adulthood. Domain specific changes include greater recruitment of frontal brain regions during complex cognitive tasks, reduced hemispheric lateralization in anterior brain regions [e.g. 80,94] and evidence for enhanced activation in regions typically engaged by young adults [68-70]. Taken together, these domain specific patterns of agerelated brain changes reflect a global pattern of neural dedifferentiation, suggesting older adults have reduced capacity to recruit specialized neural circuits associated with discreet processing operations [93]. Leading theories of functional brain aging generally converge around the notion that these patterns of functional brain change are compensatory. Functional dedifferentiation reflects recruitment of additional neural resources, or scaffolds, to overcome the challenges posed by degraded neural signaling and communication associated with structural brain changes in later life. However, compensation comes at the cost of neural efficiency, with older adults expending greater neural resources per unit of cognitive output [c.f. 91]. Finally, as with investigations of structural brain changes in older adulthood, researchers are increasingly studying age-related functional changes through the lens of distributed brain networks. Paralleling changes in regional activity, functional brain networks also appear to follow a dedifferentiation pattern in older adulthood, both with respect to the global network architecture of the brain and within more domain-specific cognitive networks. These network level changes are marked by reduced withinnetwork and increased between-network connections, as well as reduced modulation of network dynamics in response to changing task demands.

\section{CONCLUSIONS AND NEW FRONTIERS}

\subsection{Summary of structural and functional brain changes}

Over the last century we have learned much about the aging brain. With advances in in vivo imaging techniques we now know that the cranial vault masks profound changes that occur in the structure of the brain across the adult lifespan. By some estimates these changes may represent volume losses $0.3 \%$ per year through middle age, accelerating to almost $0.6 \%$ per year in older adulthood. We have also learned that these changes are not uniform across the cerebrum. The most profound and rapid losses occur in anterior and heteromodal association cortices, regions that are the last to reach maturation in early adulthood. Further, not all tissue compartments undergo similar rates of decline. While earlier studies suggested that white matter was not impacted in normal aging, more recent work demonstrates that changes in the volume, integrity, and overall health of the brain's white matter may be the most significant predictor of the transition from normal aging to neurodegenerative disease. Perhaps most surprising given the extent and pace of structural brain change, is the relative paucity of evidence linking these changes to cognitive abilities in later life. A central factor in this incongruity may be the role of altered brain function in mediating the impact of structural changes. Functional brain changes include both domain-specific and non-specific alterations in neural activation patterns that together point to a generalized pattern of neural dedifferentiation. Theories of neurocognitive aging, while differing somewhat with respect to specific causes and consequences, coalesce around the idea that older adults recruit additional neural resources to sustain cognitive output at a level equivalent to young, but at the cost of neural efficiency. Finally, we observed a growing trend in the literature to consider brain aging not only in terms of local changes, but as alterations in large-scale and spatially distributed brain networks. Network patterns appear to mirror local changes, with the greatest declines observed in nodal connections involving anterior and heteromodal association cortices as well as an age-related shift towards a more dedifferentiated functional network architecture.

\subsection{Emerging challenges and opportunities}


Aging brain structure and function

Despite significant advances in our understanding of the aging brain many challenges remain. Foremost among these is characterizing the interdependencies between structural and functional brain changes across the lifespan, and how these interactions influence the trajectory of age-related cognitive decline. In our review we addressed structure and function separately, mirroring the vast majority of the research literature in the field. However, mapping the interactions and contingencies between changes in brain structure and function across the lifespan is almost certainly a precondition for developing predictive biomarkers that can reliably differentiate healthy versus pathological brain aging [see 128, for a recent effort to develop an integrated computational model of senescence across the lifespan]. Advances in multivariate and machine learning analytical tools are now opening the door for the inclusion of an array of structural and functional brain metrics in a single analytical model to predict the trajectory of cognitive aging. These methods are allowing researchers to move beyond characterizing group differences to pursue the development of person-specific biomarkers of age-related cognitive change. However, progress in this direction will require large-scale studies to develop normative datasets, a daunting challenge given that variability is increasingly seen as a hallmark of neurocognitive aging [129].

The vast majority of research in the field still consists of cross-sectional, extreme group designs. As discussed above, this approach can mask individual differences, and tends to underestimate the extent of age-related change. Large-scale longitudinal studies, such as the Betula Project, the Baltimore Longitudinal Aging Study or Singapore-Longitudinal Aging Brain Study are beginning to address this issue. Further, open data initiatives are allowing for the aggregation of brain data across studies and centers resulting in unprecedented sample sizes (e.g. Human Connectome, UK Biobank projects). Combined with exponential advances in computational resources, these efforts hold significant promise for overcoming challenges posed by heterogeneity to drive the development of person-specific biomarkers, mapping structural and functional brain changes to individual trajectories of cognitive aging.

Finally, while big data initiatives hold considerable promise for biomarker development, efforts to develop a more mechanistic understanding of structural and functional brain aging are also informing the design of targeted interventions to alter the course of cognitive aging. We have drawn upon the neuromodulation theory of functional brain aging $[104,130]$ to develop a targeted behavioral intervention protocol to enhance goal-directed modulation of brain activity, and executive control capacity, in older adulthood [131]. Researchers are also drawing from network neuroscience models of brain aging to guide neurostimulation interventions to alleviate symptoms of psychiatric and neurological diseases, including diseases of aging. In one recent report, brain network analyses were used to detect regions on the cortical surface that were functionally connected to subcortical brain structures typically targeted in deep brain stimulation treatments [132]. These analyses open the possibility of stimulating surface nodes (e.g. using TMS) to activate, or suppress subcortical nodes non-invasively to alleviate symptoms of neurologic or psychiatrics disorders. While only two of many examples, these reports highlight the potential translational implications of our increasingly sophisticated understanding of the aging brain. As this review has clearly demonstrated, the first one hundred years of research exploring the structural and functional brain changes that occur across the adult lifespan have proven remarkably fruitful, enhancing our knowledge of the aging brain and highlighting the limitations and inherent pitfalls inherent to human brain mapping. Undoubtedly the next century will offer myriad advances, and surprises, as we continue the quest to map the topology and trajectory of brain aging, and leverage these discoveries to sustain and enhance cognitive functioning in later life. 
Aging brain structure and function

\section{References}

1. Finger, S. (1994). Origins of neuroscience, (New York: Oxford University Press).

2. Kemper, T.L. (1994). Neuroanatomical and neuropathological changes during aging and in dementia. In Clinical neurology of aging, 2nd edition, M.L. Albert and E.J.E. Knoepfel, eds. (New York: Oxford University Press), pp. 3-67.

3. Good, C.D., Johnsrude, I.S., Ashburner, J., Henson, R.N., Friston, K.J., and Frackowiak, R.S. (2001). A voxel-based morphometric study of ageing in 465 normal adult human brains. NeuroImage 14, 21-36.

4. Flood, D.G., and Coleman, P.D. (1988). Neuron numbers and sizes in aging brain: comparisons of human, monkey, and rodent data. Neurobiol. Aging 9, 453-463.

5. Salat, D.H., Buckner, R.L., Snyder, A.Z., Greve, D.N., Desikan, R.S., Busa, E., Morris, J.C., Dale, A.M., and Fischl, B. (2004). Thinning of the cerebral cortex in aging. Cereb. Cortex 14, 721-730.

6. Rossini, P.M., Rossi, S., Babiloni, C., and Polich, J. (2007). Clinical neurophysiology of aging brain: from normal aging to neurodegeneration. Prog. Neurobiol. 83, 375-400.

7. Freitas, C., Farzan, F., and Pascual-Leone, A. (2013). Assessing brain plasticity across the lifespan with transcranial magnetic stimulation: why, how, and what is the ultimate goal? Frontiers in neuroscience 7, 42 .

8. Rossi, S., Miniussi, C., Pasqualetti, P., Babiloni, C., Rossini, P.M., and Cappa, S.F. (2004). Age-related functional changes of prefrontal cortex in long-term memory: a repetitive transcranial magnetic stimulation study. J. Neurosci. 24, 7939-7944.

9. Zimerman, M., Nitsch, M., Giraux, P., Gerloff, C., Cohen, L.G., and Hummel, F.C. (2013). Neuroenhancement of the aging brain: restoring skill acquisition in old subjects. Ann. Neurol. 73, 10-15.

10. Samanez-Larkin, G.R., and Knutson, B. (2015). Decision making in the ageing brain: changes in affective and motivational circuits. Nat. Rev. Neurosci. 16, 278-289.

11. Schmitz, T.W., Spreng, R.N., and Alzheimer's Disease Neuroimaging, I. (2016). Basal forebrain degeneration precedes and predicts the cortical spread of Alzheimer's pathology. Nature communications 7, 13249.

12. Raz, N. (1996). Neuroanatomy of aging brain: Evidence from structural MRI. . In Neuroimaging II: Clinical applications, E.D. Bigler, ed. (New York: Plenum Press).

13. Raz, N. (2000). Aging of the brain and its impact on cognitive performance: Integration of structural and functional findings. In Handbook of aging and cognition II, F.I. Craik and T.A. Salthouse, eds. (Hillsdale, N.J.: Erlbaum), pp. 1-90.

14. Raz, N., and Lindenberger, U. (2011). Only time will tell: cross-sectional studies offer no solution to the age-brain-cognition triangle: comment on Salthouse (2011). Psychol. Bull. 137, 790-795.

15. Fjell, A.M., Westlye, L.T., Grydeland, H., Amlien, I., Espeseth, T., Reinvang, I., Raz, N., Dale, A.M., Walhovd, K.B., and Alzheimer Disease Neuroimaging, I. (2014). Accelerating cortical thinning: unique to dementia or universal in aging? Cereb. Cortex 24, 919-934.

16. Raz, N., Lindenberger, U., Rodrigue, K.M., Kennedy, K.M., Head, D., Williamson, A., Dahle, C., Gerstorf, D., and Acker, J.D. (2005). Regional brain changes in aging healthy adults: general trends, individual differences and modifiers. Cereb. Cortex 15, 1676-1689.

17. Wahlund, L.O., Agartz, I., Almqvist, O., Basun, H., Forssell, L., Saaf, J., and Wetterberg, L. (1990). The brain in healthy aged individuals: MR imaging. Radiology 174, 675-679.

18. Resnick, S.M., Pham, D.L., Kraut, M.A., Zonderman, A.B., and Davatzikos, C. (2003). Longitudinal magnetic resonance imaging studies of older adults: a shrinking brain. J. Neurosci. 23, 3295-3301. 
Aging brain structure and function

19. Thambisetty, M., Wan, J., Carass, A., An, Y., Prince, J.L., and Resnick, S.M. (2010). Longitudinal changes in cortical thickness associated with normal aging. NeuroImage 52, 1215-1223.

20. Shaw, M.E., Sachdev, P.S., Anstey, K.J., and Cherbuin, N. (2016). Age-related cortical thinning in cognitively healthy individuals in their 60s: the PATH Through Life study. Neurobiol. Aging 39, 202-209.

21. Storsve, A.B., Fjell, A.M., Tamnes, C.K., Westlye, L.T., Overbye, K., Aasland, H.W., and Walhovd, K.B. (2014). Differential longitudinal changes in cortical thickness, surface area and volume across the adult life span: regions of accelerating and decelerating change. J. Neurosci. 34, 8488-8498.

22. Leong, R.L., Lo, J.C., Sim, S.K., Zheng, H., Tandi, J., Zhou, J., and Chee, M.W. (2017). Longitudinal brain structure and cognitive changes over 8 years in an East Asian cohort. NeuroImage 147, 852-860.

23. Yang, Z., Wen, W., Jiang, J., Crawford, J.D., Reppermund, S., Levitan, C., Slavin, M.J., Kochan, N.A., Richmond, R.L., Brodaty, H., et al. (2016). Age-associated differences on structural brain MRI in nondemented individuals from 71 to 103 years. Neurobiol. Aging 40, 86-97.

24. Driscoll, I., Davatzikos, C., An, Y., Wu, X., Shen, D., Kraut, M., and Resnick, S.M. (2009). Longitudinal pattern of regional brain volume change differentiates normal aging from MCI. Neurology 72, 1906-1913.

25. Pfefferbaum, A., Rohlfing, T., Rosenbloom, M.J., Chu, W., Colrain, I.M., and Sullivan, E.V. (2013). Variation in longitudinal trajectories of regional brain volumes of healthy men and women (ages 10 to 85 years) measured with atlas-based parcellation of MRI. NeuroImage 65, 176-193.

26. Raz, N., Gunning, F.M., Head, D., Dupuis, J.H., McQuain, J., Briggs, S.D., Loken, W.J., Thornton, A.E., and Acker, J.D. (1997). Selective aging of the human cerebral cortex observed in vivo: differential vulnerability of the prefrontal gray matter. Cereb. Cortex 7, 268-282.

27. Sullivan, E.V., Rosenbloom, M., Serventi, K.L., and Pfefferbaum, A. (2004). Effects of age and sex on volumes of the thalamus, pons, and cortex. Neurobiol. Aging 25, 185-192.

28. Jernigan, T.L., Press, G.A., and Hesselink, J.R. (1990). Methods for measuring brain morphologic features on magnetic resonance images. Validation and normal aging. Arch. Neurol. 47, 27-32.

29. Pfefferbaum, A., Mathalon, D.H., Sullivan, E.V., Rawles, J.M., Zipursky, R.B., and Lim, K.O. (1994). A quantitative magnetic resonance imaging study of changes in brain morphology from infancy to late adulthood. Arch. Neurol. 51, 874-887.

30. Dotson, V.M., Szymkowicz, S.M., Sozda, C.N., Kirton, J.W., Green, M.L., O'Shea, A., McLaren, M.E., Anton, S.D., Manini, T.M., and Woods, A.J. (2015). Age Differences in Prefrontal Surface Area and Thickness in Middle Aged to Older Adults. Frontiers in aging neuroscience 7, 250.

31. Fjell, A.M., Westlye, L.T., Amlien, I., Espeseth, T., Reinvang, I., Raz, N., Agartz, I., Salat, D.H., Greve, D.N., Fischl, B., et al. (2009). High consistency of regional cortical thinning in aging across multiple samples. Cereb. Cortex 19, 2001-2012.

32. Fleischman, D.A., Leurgans, S., Arfanakis, K., Arvanitakis, Z., Barnes, L.L., Boyle, P.A., Han, S.D., and Bennett, D.A. (2014). Gray-matter macrostructure in cognitively healthy older persons: associations with age and cognition. Brain structure \& function 219, 20292049.

33. Walhovd, K.B., Fjell, A.M., Brewer, J., McEvoy, L.K., Fennema-Notestine, C., Hagler, D.J., Jr., Jennings, R.G., Karow, D., and Dale, A.M. (2010). Combining MR imaging, positron-emission tomography, and CSF biomarkers in the diagnosis and prognosis of Alzheimer disease. AJNR. Am. J. Neuroradiol. 31, 347-354. 
34. Ziegler, G., Dahnke, R., Jancke, L., Yotter, R.A., May, A., and Gaser, C. (2012). Brain structural trajectories over the adult lifespan. Hum. Brain Mapp. 33, 2377-2389.

35. Fjell, A.M., Westlye, L.T., Amlien, I., Tamnes, C.K., Grydeland, H., Engvig, A., Espeseth, T., Reinvang, I., Lundervold, A.J., Lundervold, A., et al. (2013). High-Expanding Cortical Regions in Human Development and Evolution Are Related to Higher Intellectual Abilities. Cereb. Cortex.

36. Pacheco, J., Goh, J.O., Kraut, M.A., Ferrucci, L., and Resnick, S.M. (2015). Greater cortical thinning in normal older adults predicts later cognitive impairment. Neurobiol. Aging 36, 903-908.

37. Scahill, R.I., Frost, C., Jenkins, R., Whitwell, J.L., Rossor, M.N., and Fox, N.C. (2003). A longitudinal study of brain volume changes in normal aging using serial registered magnetic resonance imaging. Arch. Neurol. 60, 989-994.

38. McGinnis, S.M., Brickhouse, M., Pascual, B., and Dickerson, B.C. (2011). Age-related changes in the thickness of cortical zones in humans. Brain Topogr. 24, 279-291.

39. Shaw, L.M., Vanderstichele, H., Knapik-Czajka, M., Clark, C.M., Aisen, P.S., Petersen, R.C., Blennow, K., Soares, H., Simon, A., Lewczuk, P., et al. (2009). Cerebrospinal fluid biomarker signature in Alzheimer's disease neuroimaging initiative subjects. Ann. Neurol. 65, 403-413.

40. Piguet, O., Double, K.L., Kril, J.J., Harasty, J., Macdonald, V., McRitchie, D.A., and Halliday, G.M. (2009). White matter loss in healthy ageing: a postmortem analysis. Neurobiol. Aging 30, 1288-1295.

41. Walhovd, K.B., Westlye, L.T., Amlien, I., Espeseth, T., Reinvang, I., Raz, N., Agartz, I., Salat, D.H., Greve, D.N., Fischl, B., et al. (2011). Consistent neuroanatomical age-related volume differences across multiple samples. Neurobiol. Aging 32, 916-932.

42. Raz, N., Ghisletta, P., Rodrigue, K.M., Kennedy, K.M., and Lindenberger, U. (2010). Trajectories of brain aging in middle-aged and older adults: regional and individual differences. NeuroImage 51, 501-511.

43. Ziegler, D.A., Piguet, O., Salat, D.H., Prince, K., Connally, E., and Corkin, S. (2010). Cognition in healthy aging is related to regional white matter integrity, but not cortical thickness. Neurobiol. Aging 31, 1912-1926.

44. Maillard, P., Crivello, F., Dufouil, C., Tzourio-Mazoyer, N., Tzourio, C., and Mazoyer, B. (2009). Longitudinal follow-up of individual white matter hyperintensities in a large cohort of elderly. Neuroradiology 51, 209-220.

45. Arvanitakis, Z., Fleischman, D.A., Arfanakis, K., Leurgans, S.E., Barnes, L.L., and Bennett, D.A. (2016). Association of white matter hyperintensities and gray matter volume with cognition in older individuals without cognitive impairment. Brain structure \& function 221, 2135-2146.

46. Hugenschmidt, C.E., Peiffer, A.M., Kraft, R.A., Casanova, R., Deibler, A.R., Burdette, J.H., Maldjian, J.A., and Laurienti, P.J. (2008). Relating imaging indices of white matter integrity and volume in healthy older adults. Cereb. Cortex 18, 433-442.

47. Bennett, I.J., and Madden, D.J. (2014). Disconnected aging: cerebral white matter integrity and age-related differences in cognition. Neuroscience 276, 187-205.

48. Bennett, I.J., Madden, D.J., Vaidya, C.J., Howard, D.V., and Howard, J.H., Jr. (2010). Agerelated differences in multiple measures of white matter integrity: A diffusion tensor imaging study of healthy aging. Hum. Brain Mapp. 31, 378-390.

49. Raz, N., Rodrigue, K.M., Kennedy, K.M., and Acker, J.D. (2007). Vascular health and longitudinal changes in brain and cognition in middle-aged and older adults. Neuropsychology 21, 149-157.

50. Yarchoan, M., Xie, S.X., Kling, M.A., Toledo, J.B., Wolk, D.A., Lee, E.B., Van Deerlin, V., Lee, V.M., Trojanowski, J.Q., and Arnold, S.E. (2012). Cerebrovascular atherosclerosis 
Aging brain structure and function

correlates with Alzheimer pathology in neurodegenerative dementias. Brain 135, 37493756.

51. Snowdon, D.A. (1997). Aging and Alzheimer's disease: lessons from the Nun Study. Gerontologist 37, 150-156.

52. Alexander-Bloch, A., Giedd, J.N., and Bullmore, E. (2013). Imaging structural co-variance between human brain regions. Nat. Rev. Neurosci. 14, 322-336.

53. Evans, A.C. (2013). Networks of anatomical covariance. NeuroImage 80, 489-504.

54. Mechelli, A., Friston, K.J., Frackowiak, R.S., and Price, C.J. (2005). Structural covariance in the human cortex. J. Neurosci. 25, 8303-8310.

55. Andrews, T.J., Halpern, S.D., and Purves, D. (1997). Correlated size variations in human visual cortex, lateral geniculate nucleus, and optic tract. J. Neurosci. 17, 2859-2868.

56. Alexander, G.E., Chen, K., Merkley, T.L., Reiman, E.M., Caselli, R.J., Aschenbrenner, M., Santerre-Lemmon, L., Lewis, D.J., Pietrini, P., Teipel, S.J., et al. (2006). Regional network of magnetic resonance imaging gray matter volume in healthy aging. Neuroreport $17,951-$ 956.

57. Brickman, A.M., Habeck, C., Zarahn, E., Flynn, J., and Stern, Y. (2007). Structural MRI covariance patterns associated with normal aging and neuropsychological functioning. Neurobiol. Aging 28, 284-295.

58. Chen, Z.J., He, Y., Rosa-Neto, P., Gong, G., and Evans, A.C. (2011). Age-related alterations in the modular organization of structural cortical network by using cortical thickness from MRI. NeuroImage 56, 235-245.

59. DuPre, E., and Spreng, R.N. (In press). Structural covariance networks across the lifespan, from $6-94$ years of age. Network Neuroscience.

60. Meunier, D., Achard, S., Morcom, A., and Bullmore, E. (2009). Age-related changes in modular organization of human brain functional networks. NeuroImage 44, 715-723.

61. Montembeault, M., Joubert, S., Doyon, J., Carrier, J., Gagnon, J.F., Monchi, O., Lungu, O., Belleville, S., and Brambati, S.M. (2012). The impact of aging on gray matter structural covariance networks. NeuroImage 63, 754-759.

62. Spreng, R.N., and Turner, G.R. (2013). Structural covariance of the default network in healthy and pathological aging. J. Neurosci. 33, 15226-15234.

63. Zhao, T., Cao, M., Niu, H., Zuo, X.N., Evans, A., He, Y., Dong, Q., and Shu, N. (2015). Age-related changes in the topological organization of the white matter structural connectome across the human lifespan. Hum. Brain Mapp. 36, 3777-3792.

64. Wu, K., Taki, Y., Sato, K., Kinomura, S., Goto, R., Okada, K., Kawashima, R., He, Y., Evans, A.C., and Fukuda, H. (2012). Age-related changes in topological organization of structural brain networks in healthy individuals. Hum. Brain Mapp. 33, 552-568.

65. Andrews-Hanna, J.R., Smallwood, J., and Spreng, R.N. (2014). The default network and self-generated thought: component processes, dynamic control, and clinical relevance. Ann. N. Y. Acad. Sci. 1316, 29-52.

66. Seeley, W.W., Crawford, R.K., Zhou, J., Miller, B.L., and Greicius, M.D. (2009). Neurodegenerative diseases target large-scale human brain networks. Neuron 62, 42-52.

67. Steffener, J., Brickman, A.M., Habeck, C.G., Salthouse, T.A., and Stern, Y. (2013). Cerebral blood flow and gray matter volume covariance patterns of cognition in aging. Hum. Brain Mapp. 34, 3267-3279.

68. Spreng, R.N., Wojtowicz, M., and Grady, C.L. (2010). Reliable differences in brain activity between young and old adults: a quantitative meta-analysis across multiple cognitive domains. Neurosci. Biobehav. Rev. 34, 1178-1194.

69. Spreng, R.N., Shoemaker, L., and Turner, G.R. (2017). Executive functions and neurocognitive aging. In Executive functions in heatlh and disease, E. Goldberg, ed. (San Diego, CA, U.S.A.: Elsevier). 
Aging brain structure and function

70. Turner, G.R., and Spreng, R.N. (2012). Executive functions and neurocognitive aging: dissociable patterns of brain activity. Neurobiol. Aging 33, 826 e821-813.

71. Grady, C.L. (2012). The cognitive neuroscience of ageing. Nat. Rev. Neurosci. 13, 491505 .

72. Grady, C.L. (2008). Cognitive neuroscience of aging. Ann. N. Y. Acad. Sci. 1124, 127144.

73. Greenwood, P.M. (2007). Functional plasticity in cognitive aging: review and hypothesis. Neuropsychology 21, 657-673.

74. Hedden, T., and Gabrieli, J.D. (2004). Insights into the ageing mind: a view from cognitive neuroscience. Nat. Rev. Neurosci. 5, 87-96.

75. Park, D.C., and Reuter-Lorenz, P. (2009). The adaptive brain: aging and neurocognitive scaffolding. Annu. Rev. Psychol. 60, 173-196.

76. Reuter-Lorenz, P.A., and Lustig, C. (2005). Brain aging: reorganizing discoveries about the aging mind. Curr. Opin. Neurobiol. 15, 245-251.

77. Reuter-Lorenz, P.A., and Park, D.C. (2014). How does it STAC up? Revisiting the scaffolding theory of aging and cognition. Neuropsychol. Rev. 24, 355-370.

78. Park, D.C., Polk, T.A., Mikels, J.A., Taylor, S.F., and Marshuetz, C. (2001). Cerebral aging: integration of brain and behavioral models of cognitive function. Dialogues in clinical neuroscience 3, 151-165.

79. Grady, C.L., Maisog, L.M., Horwitz, B., Ungerleider, L.G., Mentis, M.J., Salerno, J.A., Pietrini, P., Wagner, E., and Haxby, J.V. (1994). Age-related changes in cortical blood flow activation during visual processing of faces and location. J. Neurosci. 14, 1450-1462.

80. Cabeza, R. (2002). Hemispheric asymmetry reduction in older adults: the HAROLD model. Psychol. Aging 17, 85-100.

81. Cabeza, R., Anderson, N.D., Locantore, J.K., and McIntosh, A.R. (2002). Aging gracefully: compensatory brain activity in high-performing older adults. NeuroImage 17, 1394-1402.

82. Grady, C.L. (1996). Age-related changes in cortical blood flow activation during perception and memory. Ann. N. Y. Acad. Sci. 777, 14-21.

83. McIntosh, A.R. (1999). Mapping cognition to the brain through neural interactions. Memory 7, 523-548.

84. Velanova, K., Lustig, C., Jacoby, L.L., and Buckner, R.L. (2007). Evidence for frontally mediated controlled processing differences in older adults. Cereb. Cortex 17, 1033-1046.

85. Madden, D.J., Spaniol, J., Whiting, W.L., Bucur, B., Provenzale, J.M., Cabeza, R., White, L.E., and Huettel, S.A. (2007). Adult age differences in the functional neuroanatomy of visual attention: a combined fMRI and DTI study. Neurobiol. Aging 28, 459-476.

86. Cappell, K.A., Gmeindl, L., and Reuter-Lorenz, P.A. (2010). Age differences in prefontal recruitment during verbal working memory maintenance depend on memory load. Cortex 46, 462-473.

87. Mattay, V.S., Fera, F., Tessitore, A., Hariri, A.R., Berman, K.F., Das, S., MeyerLindenberg, A., Goldberg, T.E., Callicott, J.H., and Weinberger, D.R. (2006). Neurophysiological correlates of age-related changes in working memory capacity. Neurosci. Lett. 392, 32-37.

88. Reuter-Lorenz, Jonides, J., Smith, E.E., Hartley, A., Miller, A., Marshuetz, C., and Koeppe, R.A. (2000). Age differences in the frontal lateralization of verbal and spatial working memory revealed by PET. J. Cogn. Neurosci. 12, 174-187.

89. Rypma, B., and D'Esposito, M. (2000). Isolating the neural mechanisms of age-related changes in human working memory. Nat. Neurosci. 3, 509-515.

90. Colcombe, S.J., Kramer, A.F., Erickson, K.I., and Scalf, P. (2005). The implications of cortical recruitment and brain morphology for individual differences in inhibitory function in aging humans. Psychol. Aging 20,363-375. 
91. Zarahn, E., Rakitin, B., Abela, D., Flynn, J., and Stern, Y. (2007). Age-related changes in brain activation during a delayed item recognition task. Neurobiol. Aging 28, 784-798.

92. Craik, F.I., and Bialystok, E. (2006). Cognition through the lifespan: mechanisms of change. Trends in cognitive sciences 10,131-138.

93. Li, S.C., Lindenberger, U., and Sikstrom, S. (2001). Aging cognition: from neuromodulation to representation. Trends in cognitive sciences 5, 479-486.

94. Grady, C.L., Maisog, J.M., Horwitz, B., Ungerleider, L.G., Mentis, M.J., Salerno, J.A., Pietrini, P., Wagner, E., and Haxby, J.V. (1994). Age-related changes in cortical blood flow activation during visual processing of faces and location. J. Neurosci. 14, 1450-1462.

95. Davis, S.W., Dennis, N.A., Daselaar, S.M., Fleck, M.S., and Cabeza, R. (2008). Que PASA? The posterior-anterior shift in aging. Cereb. Cortex 18, 1201-1209.

96. Rieck, J.R., Rodrigue, K.M., Boylan, M.A., and Kennedy, K.M. (2017). Age-related reduction of BOLD modulation to cognitive difficulty predicts poorer task accuracy and poorer fluid reasoning ability. NeuroImage 147, 262-271.

97. Turner, G.R., and Spreng, R.N. (2015). Prefrontal Engagement and Reduced Default Network Suppression Co-occur and Are Dynamically Coupled in Older Adults: The Default-Executive Coupling Hypothesis of Aging. J. Cogn. Neurosci. 27, 2462-2476.

98. Gutchess, A.H., Welsh, R.C., Hedden, T., Bangert, A., Minear, M., Liu, L.L., and Park, D.C. (2005). Aging and the neural correlates of successful picture encoding: frontal activations compensate for decreased medial-temporal activity. J. Cogn. Neurosci. 17, 8496.

99. Baltes, P.B., and Lindenberger, U. (1997). Emergence of a powerful connection between sensory and cognitive functions across the adult life span: a new window to the study of cognitive aging? Psychol. Aging 12, 12-21.

100. Cabeza, R., Daselaar, S.M., Dolcos, F., Prince, S.E., Budde, M., and Nyberg, L. (2004). Task-independent and task-specific age effects on brain activity during working memory, visual attention and episodic retrieval. Cereb. Cortex 14, 364-375.

101. Reuter-Lorenz, P., and Cappell, K.A. (2008). Neurocognitive Aging and the Compensation Hypothesis. Current Directions in Psychological Science 17, 177-182.

102. Li, S.C., and Rieckmann, A. (2014). Neuromodulation and aging: implications of aging neuronal gain control on cognition. Curr. Opin. Neurobiol. 29, 148-158.

103. Gazzaley, A., Cooney, J.W., Rissman, J., and D'Esposito, M. (2005). Top-down suppression deficit underlies working memory impairment in normal aging. Nat. Neurosci. 8, 1298-1300.

104. Gazzaley, A., and D'Esposito, M. (2007). Top-down modulation and normal aging. Ann. N. Y. Acad. Sci. 1097, 67-83.

105. Payer, D., Marshuetz, C., Sutton, B., Hebrank, A., Welsh, R.C., and Park, D.C. (2006). Decreased neural specialization in old adults on a working memory task. Neuroreport 17 , 487-491.

106. Schmitz, T.W., Dixon, M.L., Anderson, A.K., and De Rosa, E. (2014). Distinguishing attentional gain and tuning in young and older adults. Neurobiol. Aging 35, 2514-2525.

107. Damoiseaux, J.S. (2017). Effects of aging on functional and structural brain connectivity. NeuroImage.

108. Andrews-Hanna, J.R., Snyder, A.Z., Vincent, J.L., Lustig, C., Head, D., Raichle, M.E., and Buckner, R.L. (2007). Disruption of large-scale brain systems in advanced aging. Neuron 56, 924-935.

109. Damoiseaux, J.S., Beckmann, C.F., Arigita, E.J., Barkhof, F., Scheltens, P., Stam, C.J., Smith, S.M., and Rombouts, S.A. (2008). Reduced resting-state brain activity in the "default network" in normal aging. Cereb. Cortex 18, 1856-1864.

110. Grady, C.L., Protzner, A.B., Kovacevic, N., Strother, S.C., Afshin-Pour, B., Wojtowicz, M., Anderson, J.A., Churchill, N., and McIntosh, A.R. (2010). A multivariate analysis of 
age-related differences in default mode and task-positive networks across multiple cognitive domains. Cereb. Cortex 20, 1432-1447.

111. Hafkemeijer, A., van der Grond, J., and Rombouts, S.A. (2012). Imaging the default mode network in aging and dementia. Biochim. Biophys. Acta 1822, 431-441.

112. Persson, J., Lustig, C., Nelson, J.K., and Reuter-Lorenz, P.A. (2007). Age differences in deactivation: a link to cognitive control? J. Cogn. Neurosci. 19, 1021-1032.

113. Sambataro, F., Murty, V.P., Callicott, J.H., Tan, H.Y., Das, S., Weinberger, D.R., and Mattay, V.S. (2010). Age-related alterations in default mode network: impact on working memory performance. Neurobiol. Aging 31, 839-852.

114. Sheline, Y.I., and Raichle, M.E. (2013). Resting State Functional Connectivity in Preclinical Alzheimer's Disease. Biol. Psychiatry.

115. Buckner, R.L., Andrews-Hanna, J.R., and Schacter, D.L. (2008). The brain's default network: anatomy, function, and relevance to disease. Ann. N. Y. Acad. Sci. 1124, 1-38.

116. Hansen, N.L., Lauritzen, M., Mortensen, E.L., Osler, M., Avlund, K., Fagerlund, B., and Rostrup, E. (2014). Subclinical cognitive decline in middle-age is associated with reduced task-induced deactivation of the brain's default mode network. Hum. Brain Mapp. 35, 4488-4498.

117. Lustig, C., Snyder, A.Z., Bhakta, M., O'Brien, K.C., McAvoy, M., Raichle, M.E., Morris, J.C., and Buckner, R.L. (2003). Functional deactivations: change with age and dementia of the Alzheimer type. Proc. Natl. Acad. Sci. U. S. A. 100, 14504-14509.

118. Geerligs, L., Maurits, N.M., Renken, R.J., and Lorist, M.M. (2014). Reduced specificity of functional connectivity in the aging brain during task performance. Hum. Brain Mapp. 35, 319-330.

119. Chan, M.Y., Park, D.C., Savalia, N.K., Petersen, S.E., and Wig, G.S. (2014). Decreased segregation of brain systems across the healthy adult lifespan. Proc. Natl. Acad. Sci. U. S. A. 111, E4997-5006.

120. Geerligs, L., Renken, R.J., Saliasi, E., Maurits, N.M., and Lorist, M.M. (2015). A BrainWide Study of Age-Related Changes in Functional Connectivity. Cereb. Cortex 25, 19871999.

121. Muller, A.M., Merillat, S., and Jancke, L. (2015). Older but still fluent? Insights from the intrinsically active baseline configuration of the aging brain using a data driven graphtheoretical approach. NeuroImage 127, 346-362.

122. Spreng, R.N., Stevens, W.D., Viviano, J.D., and Schacter, D.L. (2016). Attenuated anticorrelation between the default and dorsal attention networks with aging: evidence from task and rest. Neurobiol. Aging 45, 149-160.

123. Grady, C., Sarraf, S., Saverino, C., and Campbell, K. (2016). Age differences in the functional interactions among the default, frontoparietal control, and dorsal attention networks. Neurobiol. Aging 41, 159-172.

124. Spreng, R.N., and Schacter, D.L. (2012). Default Network Modulation and Large-Scale Network Interactivity in Healthy Young and Old Adults. Cereb. Cortex 22, 2610-2621.

125. Gallen, C.L., Turner, G.R., Adnan, A., and D'Esposito, M. (2016). Reconfiguration of brain network architecture to support executive control in aging. Neurobiol. Aging 44, 42-52.

126. Spreng, R.N., Lockrow, A.W., DuPre, E., Setton, R., Spreng, K.A.P., and Turner, G.R. (2017). Semanticized autobiographical memory and the default - executive coupling hypothesis of aging. Neuropsychologia.

127. Ng, K.K., Lo, J.C., Lim, J.K., Chee, M.W., and Zhou, J. (2016). Reduced functional segregation between the default mode network and the executive control network in healthy older adults: A longitudinal study. NeuroImage 133, 321-330.

128. Naik, S., Banerjee, A., Bapi, R.S., Deco, G., and Roy, D. (2017). Metastability in Senescence. Trends in cognitive sciences 21, 509-521. 
129. Garrett, D.D., Samanez-Larkin, G.R., MacDonald, S.W., Lindenberger, U., McIntosh, A.R., and Grady, C.L. (2013). Moment-to-moment brain signal variability: a next frontier in human brain mapping? Neurosci. Biobehav. Rev. 37, 610-624.

130. D'Esposito, M., and Chen, A.J. (2006). Neural mechanisms of prefrontal cortical function: implications for cognitive rehabilitation. Prog. Brain Res. 157, 123-139.

131. Adnan, A., Chen, A.J.W., Novakovic-Agopian, T., D'Esposito, M., and Turner, G.R. (2017). Brain Changes Following Executive Control Training in Older Adults. Neurorehabil Neural Repair, 1545968317728580.

132. Fox, M.D., Buckner, R.L., Liu, H., Chakravarty, M.M., Lozano, A.M., and Pascual-Leone, A. (2014). Resting-state networks link invasive and noninvasive brain stimulation across diverse psychiatric and neurological diseases. Proc. Natl. Acad. Sci. U. S. A. 111, E43674375 . 